\title{
Investigation of a non-linear system under partially prescribed random excitation
}

\author{
R.N. Iyengar*, Bisakha Basak \\ Department of Civil Engineering, Indian Institute of Science, Bangalore 560012, India
}

\begin{abstract}
The problem of non-linear systems excited by random forces with known power spectral density functions and unspecified probability structure is considered. Sufficient, but not necessary, conditions on the input under which the response can be a Gaussian process are investigated. The approach is illustrated by investigating the hardening spring cubic oscillator under wide and narrow band excitations. The non-Gaussian probability density of the input that leads to Gaussian response is determined.
\end{abstract}

Keywords: Non-linear oscillator; Non-Gaussian input; Inverse approach

\section{Introduction}

Exact solutions for a non-linear system under random excitation are rare. It is known that even under ideal white noise excitation, only for certain types of non-linear systems, the exact probability density function (PDF) of the response in the steady state can be obtained [1]. Usually the power spectral density (PSD) of the input is non-white and the PDF is taken to be Gaussian to seek an approximate solution through equivalent linearization techniques [2]. In this context, a system widely studied has been the viscously damped hardening cubic oscillator. The availability of an exact solution for this system under white noise

\footnotetext{
* Corresponding author.

E-mail address: rni@civil.iisc.ernet.in (R.N. Iyengar).
}

excitation helps one to understand the limitations of approximate methods. Apart from results on the stationary PDF, one would be interested in the response PSD function. In linear problems, response PSD is easily found as the product of the input PSD and the system frequency response function, for any arbitrary input PDF. Such a facility is not available with the simplest non-linear system. The stochastic averaging method [3] overcomes some of these difficulties to lead to non-Gaussian response distributions and PSD functions. However, this method is restricted to wideband excitations and lightly damped systems, such that the response can be taken to be a narrow band process. No general method is available at present to obtain the response PDF and PSD of a non-linear system under a given arbitrary Gaussian random input. With the above points in the background, a novel concept is introduced here to find a particular solution for a given 
system excited by an input with prescribed PSD but unspecified probability structure. The method investigates under what sufficient, but not necessary, conditions on the input PDF the response will be a Gaussian process. Application of this inverse approach is illustrated by investigating Duffing's oscillator, under random excitation with prescribed PSD. The probability structure of the input that leads to Gaussian response PDF is determined.

\section{Duffing's oscillator}

Duffing's oscillator with hardening spring under a stationary random excitation with zero mean and given PSD function $S_{f f}(\Omega)$ is described by

$\ddot{x}+2 \eta \omega \dot{x}+\omega^{2} x+\alpha \omega^{2} x^{3}=f(t)$.

The steady-state variance $\sigma_{1}^{2}$ can be easily found out in the linear case with $\alpha=0$. Eq. (1) can be rewritten in terms of $y=x / \sigma_{1}$ as

$\ddot{y}+2 \eta \omega \dot{y}+\omega^{2} y+\varepsilon \omega^{2} y^{3}=\frac{f(t)}{\sigma_{1}}$,

where $\varepsilon=\alpha \sigma_{1}^{2}$.

In the traditional equivalent linearization and closure techniques, the response and the fully prescribed excitation are taken to be jointly Gaussian to arrive at approximate solutions. In contrast here the intention is not a solution of the above equation under general $f(t)$. The aim is limited to find sufficient conditions on $f(t)$, partially prescribed in terms of its PSD, such that $y(t)$ can be a Gaussian process. A particular solution to this problem can be found along the following lines. Eq. (2) is recast in the form

$\ddot{y}+2 \eta \omega \dot{y}+\omega^{2} y=\left[\frac{f(t)}{\sigma_{1}}-\varepsilon \omega^{2} y^{3}\right]=z(t)$.

It is easily observed that in the steady state, the PSD of $y(t)$ has to be related to the PSD of $z(t)$ as

$S_{y y}(\Omega)=S_{z z}(\Omega)|H(\Omega)|^{2}$,

where

$|H(\Omega)|^{2}=\left[\left(\omega^{2}-\Omega^{2}\right)^{2}+(2 \eta \omega \Omega)^{2}\right]^{-1}$.

Here, $S_{y y}(\Omega)$ cannot be found since $S_{z z}(\Omega)$ is not known. However, for $y(t)$ to be a Gaussian process, it is sufficient if $z(t)$ is Gaussian. This property can be exploited to find the exact PDF of $f(t)$ for a specified $S_{f f}(\Omega)$. Since

$\frac{f}{\sigma_{1}}=z+\varepsilon \omega^{2} y^{3}$,

it follows that

$$
\begin{aligned}
\left\langle f\left(t_{1}\right) f\left(t_{2}\right)\right\rangle= & \left\langle z\left(t_{1}\right) z\left(t_{2}\right)\right\rangle+\varepsilon \omega^{2}\left\langle y^{3}\left(t_{1}\right) z\left(t_{2}\right)\right\rangle \\
& +\varepsilon \omega^{2}\left\langle z\left(t_{1}\right) y^{3}\left(t_{2}\right)\right\rangle \\
& +\varepsilon^{2} \omega^{4}\left\langle y^{3}\left(t_{1}\right) y^{3}\left(t_{2}\right)\right\rangle .
\end{aligned}
$$

Since $(y, z)$ are jointly Gaussian, this equation reduces in the stationary regime to

$$
\begin{aligned}
\frac{R_{f f}(\tau)}{\sigma_{1}^{2}}= & R_{z z}(\tau)+3 \varepsilon \omega^{2} \sigma_{y}^{2}\left[R_{z y}(\tau)+R_{y z}(\tau)\right] \\
& +\varepsilon^{2} \omega^{4}\left[9 \sigma_{y}^{4} R_{y y}(\tau)+6 R_{y y}^{3}(\tau)\right],
\end{aligned}
$$

where $\tau=\left(t_{2}-t_{1}\right)$.

For the PSD function, one gets

$$
\begin{aligned}
\frac{S_{f f}(\Omega)}{\sigma_{1}^{2}}= & S_{z z}(\Omega)+3 \varepsilon \omega^{2} \sigma_{y}^{2}\left[S_{z y}(\Omega)+S_{y z}(\Omega)\right] \\
& +\varepsilon^{2} \omega^{4}\left[9 \sigma_{y}^{4} S_{y y}(\Omega)+6 S_{\mathrm{c}}(\Omega)\right],
\end{aligned}
$$

where

$$
\begin{aligned}
& S_{\mathrm{c}}(\Omega)=\frac{2}{\pi} \int_{0}^{\infty} R_{y y}^{3}(\tau) \cos \Omega \tau \mathrm{d} \tau, \\
& R_{y y}(\tau)=\int_{0}^{\infty} S_{y y}(\Omega) \cos \Omega \tau \mathrm{d} \Omega
\end{aligned}
$$

and

$\sigma_{y}^{2}=\int_{0}^{\infty} S_{y y}(\Omega) \mathrm{d} \Omega$.

Cross-correlation functions $R_{z y}(\tau), R_{y z}(\tau)$ can be represented in terms of $S_{z z}(\Omega)$ as

$$
\begin{aligned}
R_{z y}(\tau)= & \frac{1}{2 \omega_{\mathrm{d}}} \int_{0}^{\infty} S_{z z}(\Omega) \\
& \times\left[\operatorname { c o s } \Omega \tau \left\{\frac{\omega_{\mathrm{d}}+\Omega}{\eta^{2} \omega^{2}+\left(\omega_{\mathrm{d}}+\Omega\right)^{2}}\right.\right. \\
& \left.+\frac{\omega_{\mathrm{d}}-\Omega}{\eta^{2} \omega^{2}+\left(\omega_{\mathrm{d}}-\Omega\right)^{2}}\right\} \\
& -\sin \Omega \tau\left\{\frac{\eta \omega}{\eta^{2} \omega^{2}+\left(\omega_{\mathrm{d}}+\Omega\right)^{2}}\right. \\
& \left.\left.-\frac{\eta \omega}{\eta^{2} \omega^{2}+\left(\omega_{\mathrm{d}}-\Omega\right)^{2}}\right\}\right] \mathrm{d} \Omega,
\end{aligned}
$$




$$
\begin{aligned}
R_{y z}(\tau)= & \frac{1}{2 \omega_{\mathrm{d}}} \int_{0}^{\infty} S_{z z}(\Omega) \\
& \times\left[\operatorname { c o s } \Omega \tau \left\{\frac{\omega_{\mathrm{d}}+\Omega}{\eta^{2} \omega^{2}+\left(\omega_{\mathrm{d}}+\Omega\right)^{2}}\right.\right. \\
& \left.+\frac{\omega_{\mathrm{d}}-\Omega}{\eta^{2} \omega^{2}+\left(\omega_{\mathrm{d}}-\Omega\right)^{2}}\right\} \\
& +\sin \Omega \tau\left\{\frac{\eta \omega}{\eta^{2} \omega^{2}+\left(\omega_{\mathrm{d}}+\Omega\right)^{2}}\right. \\
& \left.\left.-\frac{\eta \omega}{\eta^{2} \omega^{2}+\left(\omega_{\mathrm{d}}-\Omega\right)^{2}}\right\}\right] \mathrm{d} \Omega
\end{aligned}
$$

with $\omega_{\mathrm{d}}=\omega \sqrt{1-\eta^{2}}$.

Hence,

$$
\begin{aligned}
{\left[R_{z y}(\tau)\right.} & \left.+R_{y z}(\tau)\right] \\
= & \omega_{\mathrm{d}}^{-1} \int_{0}^{\infty} S_{z z}(\Omega) \\
& \times\left[\operatorname { c o s } \Omega \tau \left\{\frac{\omega_{\mathrm{d}}+\Omega}{\eta^{2} \omega^{2}+\left(\omega_{\mathrm{d}}+\Omega\right)^{2}}\right.\right. \\
& \left.\left.+\frac{\omega_{\mathrm{d}}-\Omega}{\eta^{2} \omega^{2}+\left(\omega_{\mathrm{d}}-\Omega\right)^{2}}\right\}\right] \mathrm{d} \Omega .
\end{aligned}
$$

Further, the terms $S_{z y}(\Omega), S_{y z}(\Omega), S_{z z}(\Omega)$ can be expressed in terms of a single unknown function $S_{y y}(\Omega)$ as

$$
S_{z z}(\Omega)=\frac{S_{y y}(\Omega)}{|H(\Omega)|^{2}},
$$

$$
\begin{aligned}
{\left[S_{z y}(\Omega)+S_{y z}(\Omega)\right]=} & \omega_{\mathrm{d}}^{-1} \frac{S_{y y}(\Omega)}{|H(\Omega)|^{2}} \\
& \times\left[\frac{\omega_{\mathrm{d}}+\Omega}{\eta^{2} \omega^{2}+\left(\omega_{\mathrm{d}}+\Omega\right)^{2}}\right. \\
& \left.+\frac{\omega_{\mathrm{d}}-\Omega}{\eta^{2} \omega^{2}+\left(\omega_{\mathrm{d}}-\Omega\right)^{2}}\right],
\end{aligned}
$$

where $\omega_{\mathrm{d}}=\omega \sqrt{1-\eta^{2}}$.
Substitution of these expressions in Eq. (9) leads to

$$
\begin{aligned}
S_{y y}(\Omega)= & \frac{S_{f f}(\Omega)}{\sigma_{1}^{2}|H(\Omega)|^{2}\left[1+\frac{3 \varepsilon \omega^{2} \sigma_{y}^{2}}{\omega_{\mathrm{d}}}\right.} \\
& \times\left\{\frac{\omega_{\mathrm{d}}+\Omega}{\eta^{2} \omega^{2}+\left(\omega_{\mathrm{d}}+\Omega\right)^{2}}\right. \\
& \left.+\frac{\omega_{\mathrm{d}}-\Omega}{\eta^{2} \omega^{2}+\left(\omega_{\mathrm{d}}-\Omega\right)^{2}}\right\}+9 \varepsilon^{2} \omega^{4} \\
& \left.\times \sigma_{y}^{4}|H(\Omega)|^{2}+6 \varepsilon^{2} \omega^{4}|H(\Omega)|^{2} \frac{S_{\mathrm{c}}}{S_{y y}}\right]^{-1}
\end{aligned}
$$

This transcendental equation is a sufficient condition for finding the response PSD function $S_{y y}(\Omega)$, such that $y(t)$ is a Gaussian process. Once $S_{y y}(\Omega)$ is found, the joint PDF $p(z, y)$ which is Gaussian can be expressed as

$$
\begin{aligned}
p(z, y ; \tau)= & \left\{2 \pi \sigma_{z} \sigma_{y} \sqrt{1-r_{y z}^{2}(\tau)}\right\}^{-1} \\
& \times \exp \left[-\frac{1}{2\left\{1-r_{y z}^{2}(\tau)\right\}}\right. \\
& \left.\times\left\{\frac{z^{2}}{\sigma_{z}^{2}}-\frac{2 r_{y z}(\tau) z y}{\sigma_{z} \sigma_{y}}+\frac{y^{2}}{\sigma_{y}^{2}}\right\}\right],
\end{aligned}
$$

where

$$
\sigma_{z}^{2}=\int_{0}^{\infty} \frac{S_{y y}(\Omega)}{|H(\Omega)|^{2}} \mathrm{~d} \Omega, \quad r_{y z}(\tau)=\frac{R_{y z}(\tau)}{\sigma_{z} \sigma_{y}} .
$$

Now, at $\tau=0$, the correlation coefficient between $z$ and $y$ is

$$
\begin{aligned}
r_{0}= & \frac{R_{z y}(0)}{\sigma_{y} \sigma_{z}}=\frac{R_{y z}(0)}{\sigma_{y} \sigma_{z}} \\
= & \frac{1}{2 \sigma_{y} \sigma_{z} \omega_{\mathrm{d}}} \int_{0}^{\infty} S_{z z}(\Omega)\left\{\frac{\omega_{\mathrm{d}}+\Omega}{\eta^{2} \omega^{2}+\left(\omega_{\mathrm{d}}+\Omega\right)^{2}}\right. \\
& \left.+\frac{\omega_{\mathrm{d}}-\Omega}{\eta^{2} \omega^{2}+\left(\omega_{\mathrm{d}}-\Omega\right)^{2}}\right\} \mathrm{d} \Omega .
\end{aligned}
$$

Since $f$ is expressed in terms of $z$ and $y$, through Eq. (6), the first-order PDF of the excitation can be 


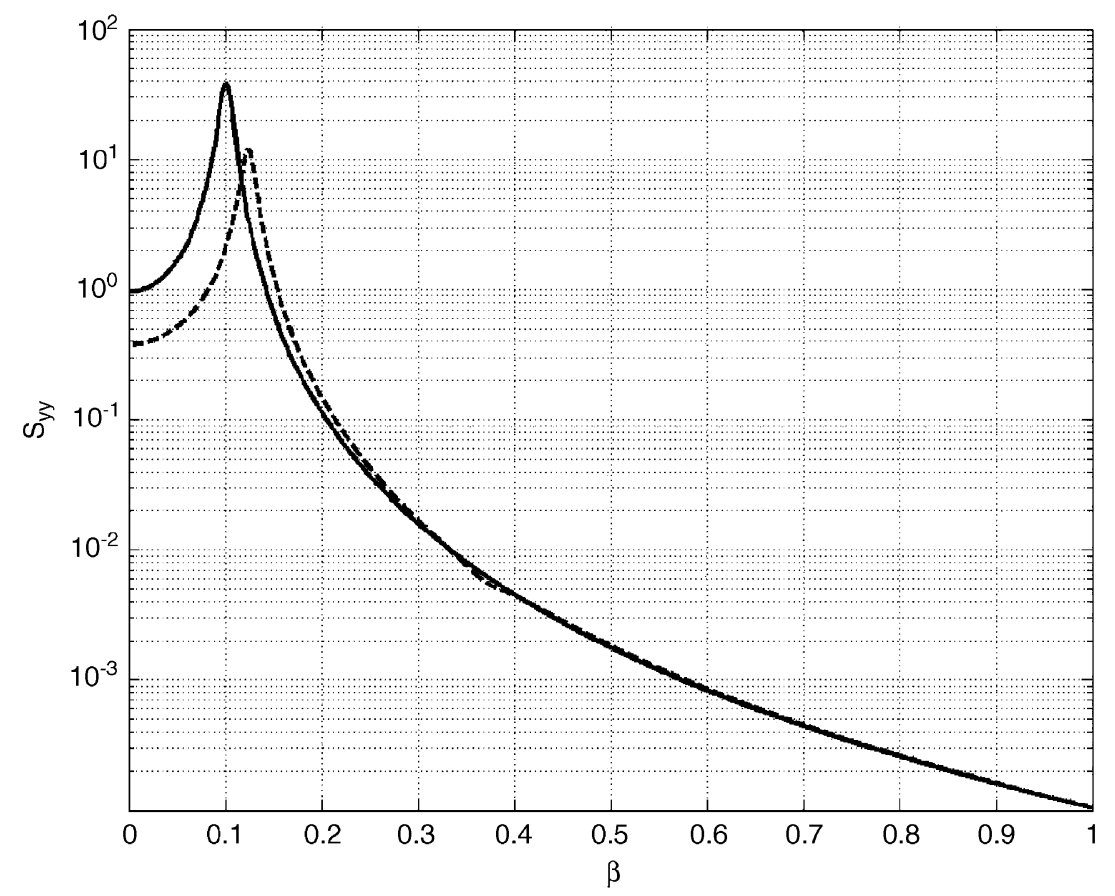

Fig. 1. PSD of $y(t) ;-\varepsilon=0.01,--\varepsilon=0.5$.

obtained by a suitable transformation on Eq. (17) as

$$
\begin{aligned}
p(u)= & \frac{\omega^{2}}{2 \pi \sigma_{z} \sigma_{y} \sqrt{1-r_{0}^{2}}} \int_{-\infty}^{\infty} \exp \left[-\frac{1}{2\left(1-r_{0}^{2}\right)}\right. \\
& \times\left\{\frac{\omega^{4}\left(u-\varepsilon v^{3}\right)^{2}}{\sigma_{z}^{2}}\right. \\
& \left.\left.-\frac{2 r_{0} \omega^{2}\left(u-\varepsilon v^{3}\right) v}{\sigma_{z} \sigma_{y}}+\frac{v^{2}}{\sigma_{y}^{2}}\right\}\right] \mathrm{d} v
\end{aligned}
$$

where $u=f / \omega^{2} \sigma_{1}$.

Higher-order PDF of the excitation process can be found with further computational effort. For this particular type of random excitation, with a specified PSD function, the response process $y(t)$ will be exactly Gaussian. Numerical results of the above analysis are presented for broad and narrow band excitations.

\section{Broadband input}

Let the input be a band-limited process, with uniform PSD $S_{0}$ and cut-off frequency $\omega_{\mathrm{c}}$ such that the variance of the input is given by

$$
\sigma_{f}^{2}=S_{0} \omega_{\mathrm{c}} .
$$

For a linear system with $\varepsilon=0$, under ideal white noise, the steady-state response variance is

$$
\sigma_{1}^{2}=\frac{\pi S_{0}}{4 \eta \omega^{3}}
$$

The non-dimensional response under the band-limited case is taken as $y=x / \sigma_{1}$ as in Eq. (2). The frequency parameters are expressed for numerical work as

$\beta=\frac{\Omega}{\omega_{\mathrm{c}}}, \quad \bar{\omega}=\frac{\omega_{\mathrm{c}}}{\omega}$.

The excitation is taken in the form $u=\left(f / \omega^{2} \sigma_{1}\right)$. For this case, Eq. (16) is solved iteratively to get the response PSD function. The results are shown in Fig. 1 for $\varepsilon=0.01$ and 0.5 , with $\eta=0.08$ and $\bar{\omega}=10$. The nonGaussian PDF of the input process $u(t)$ is shown in Fig. 2. In Fig. 3, the Gaussian response PDF results are shown. For values of $\bar{\omega} \gg 1$ and $\varepsilon \ll 1$, it is expected 


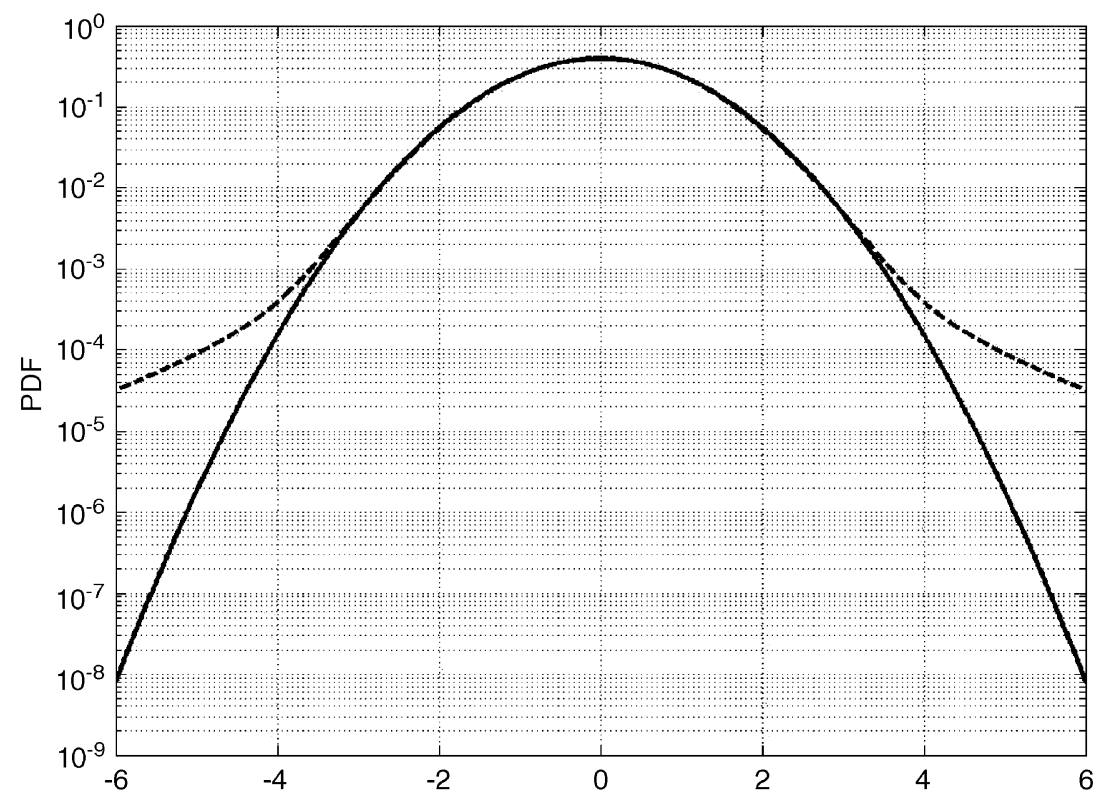

Fig. 2. PDF of $u ;-\varepsilon=0.01$, kurtosis $=3.00,--\varepsilon=0.5$, kurtosis $=3.39$.

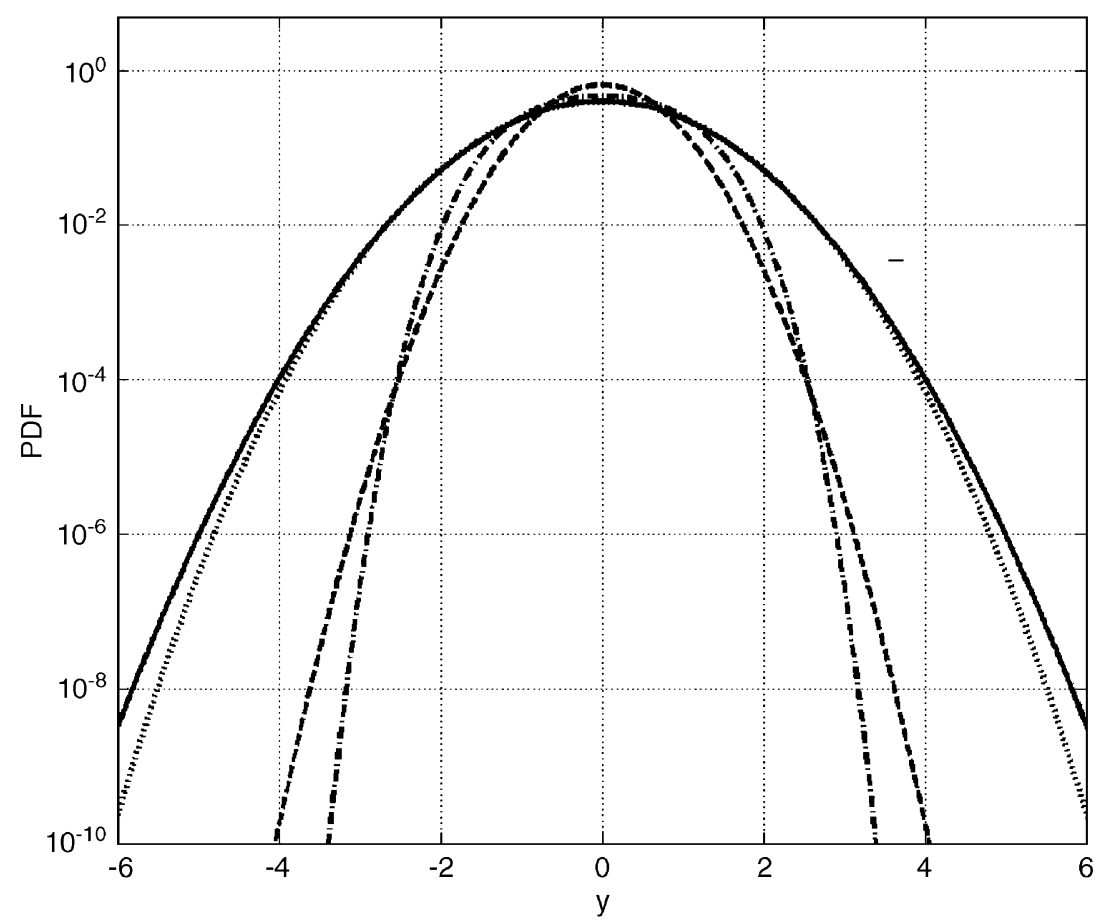

Fig. 3. PDF of $y$; - Gaussian, $\cdots$ white noise, $\varepsilon=0.01,---$ Gaussian, --- white noise, $\varepsilon=0.5$. 


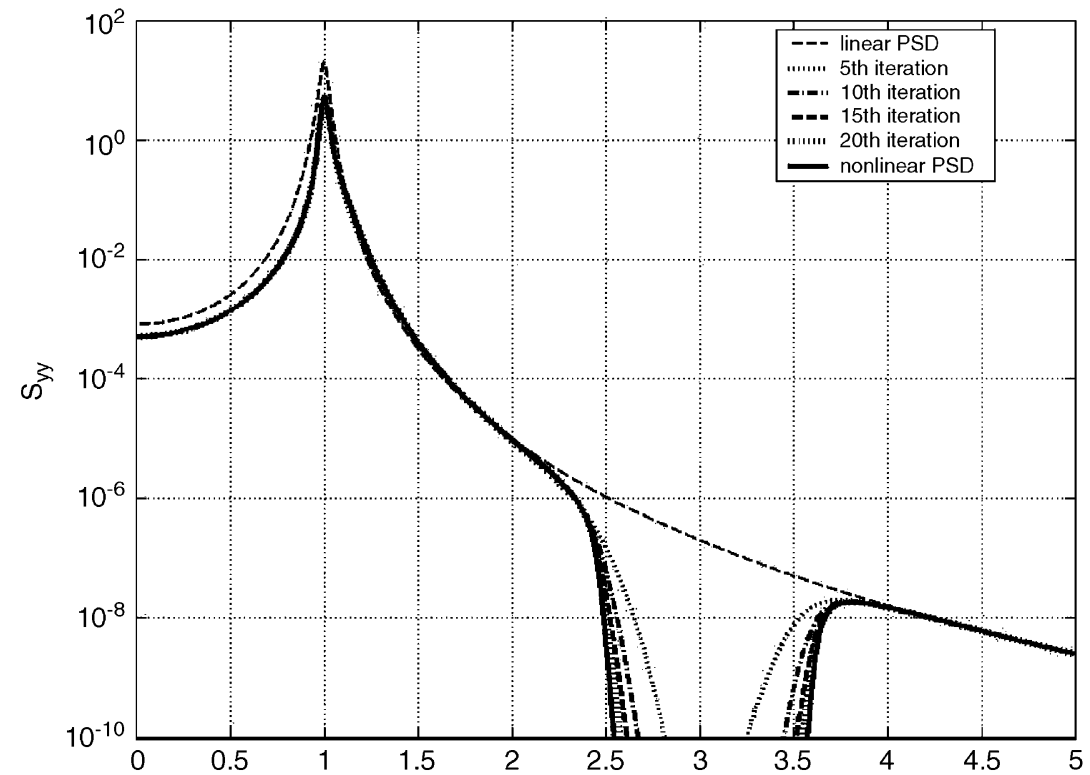

(a)
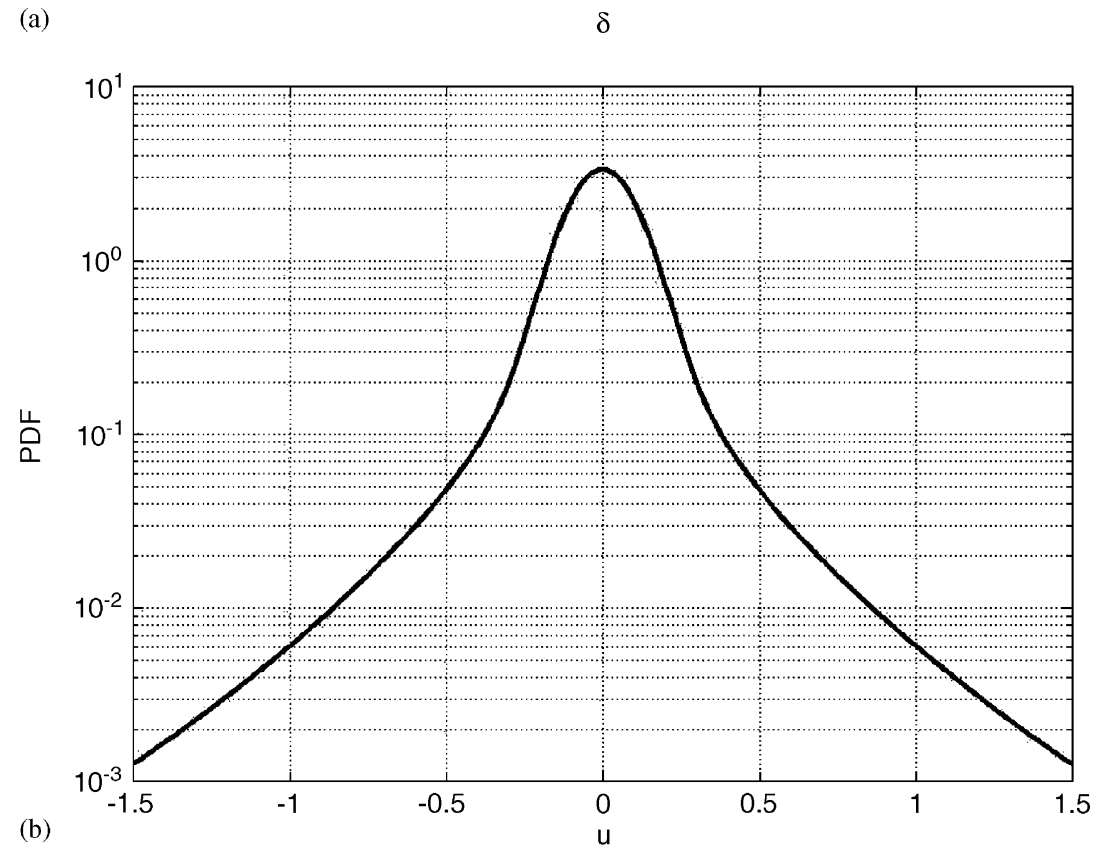

Fig. 4. (a) PSD of $y(t), \bar{\lambda}=1$. (b) PDF of $u, \bar{\lambda}=1$, kurtosis $=26.89$.

that the present results compare with the known exact solutions under ideal white noise excitation. This is seen to be true for PDF of $y$, for small values of $\varepsilon$, in Fig. 3. Similarly for this case, the nature of the inverse excitation process also re- mains nearly Gaussian as shown in Fig. 2. However, as the value of $\varepsilon$ increases, the above features change considerably and the excitation has to become strongly non-Gaussian to yield Gaussian response. 

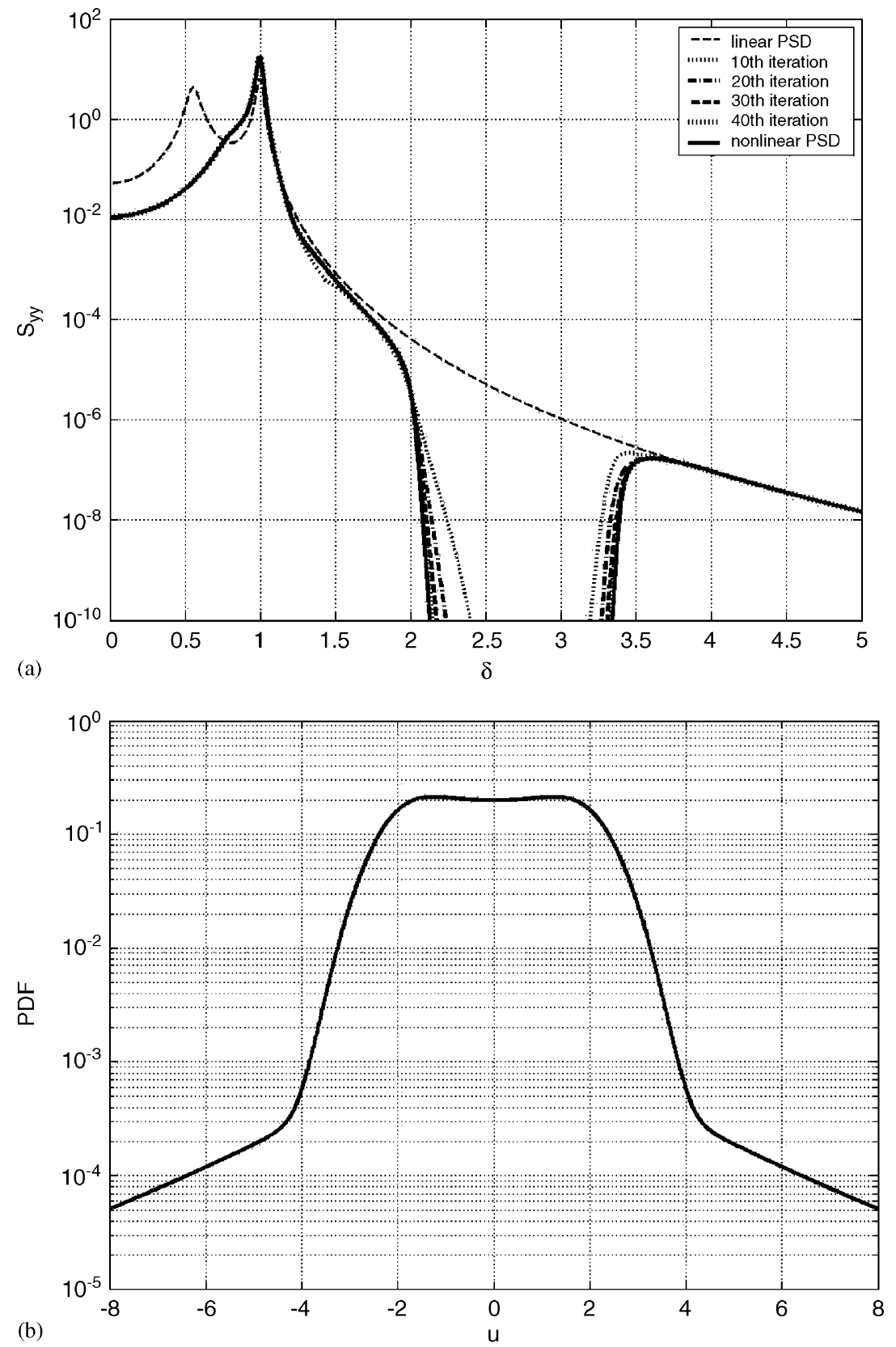

Fig. 5. (a) PSD of $y(t), \bar{\lambda}=1.8$. (b) PDF of $u, \bar{\lambda}=1.8$, kurtosis $=3.21$.

\section{Narrowband input}

As a second example of the inverse approach, the excitation in Eq. (2) is considered a random process with prescribed PSD function

$$
S_{f f}(\Omega)=\frac{S_{0}}{\left[\left(\lambda^{2}-\Omega^{2}\right)^{2}+(2 \xi \lambda \Omega)^{2}\right]} .
$$




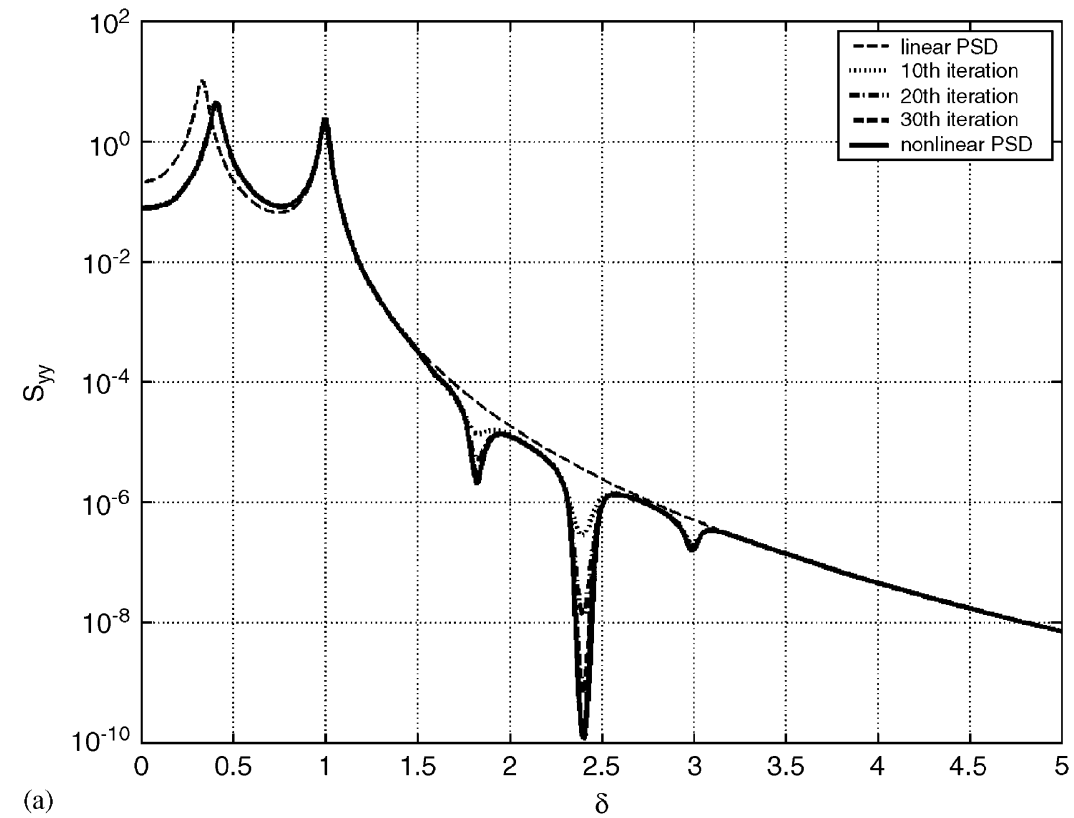

(a)

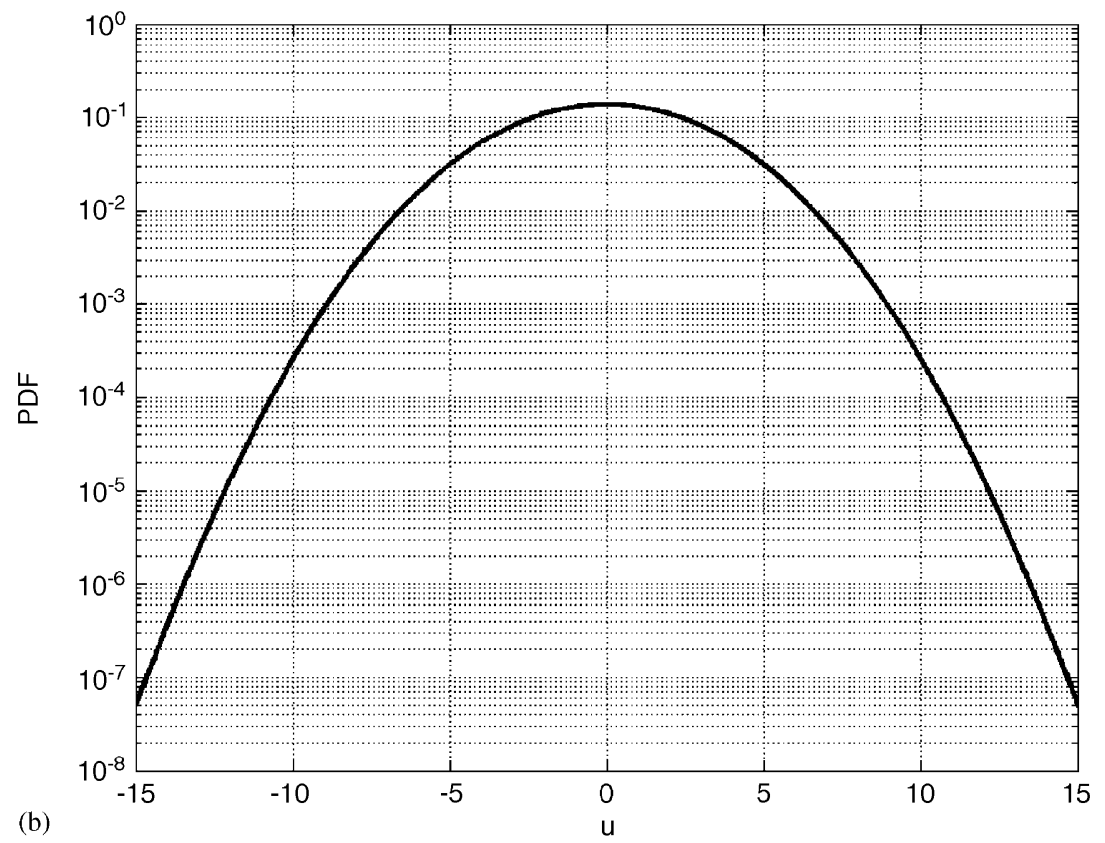

Fig. 6. (a) PSD of $y(t), \bar{\lambda}=3$. (b) PDF of $u, \bar{\lambda}=3$, kurtosis $=2.92$. 


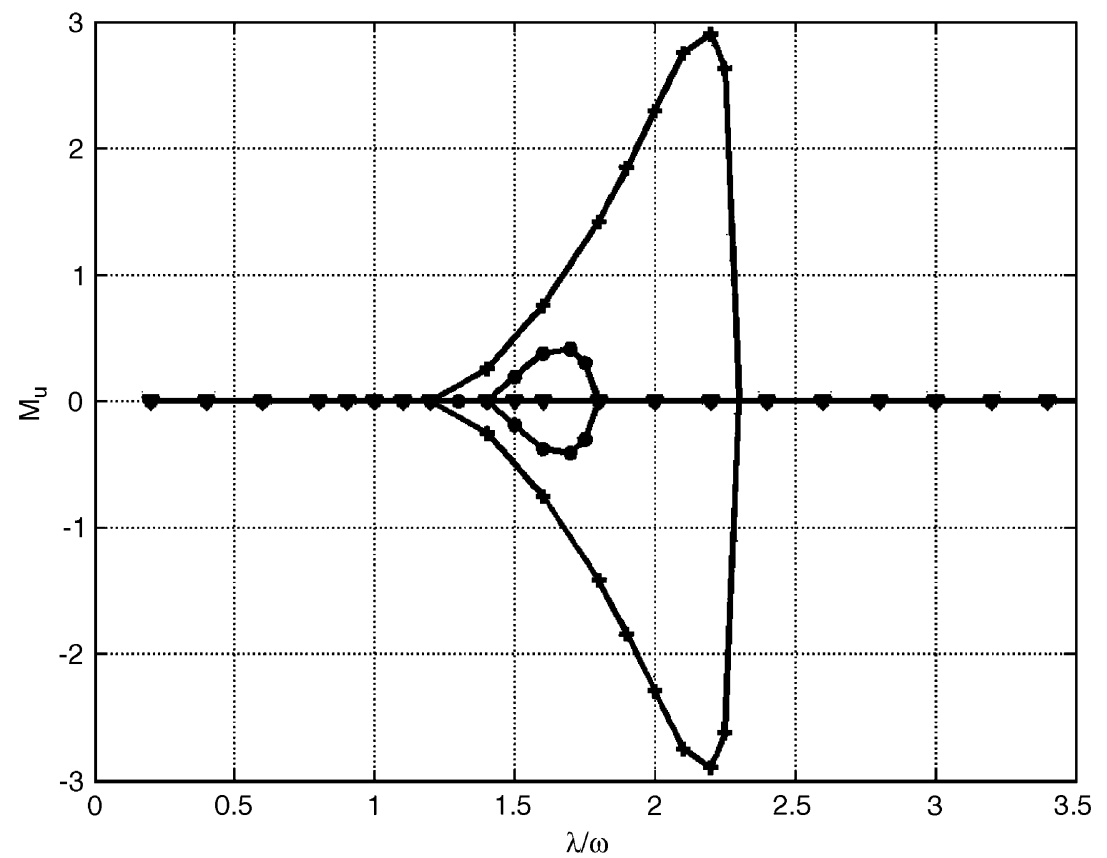

Fig. 7. Bifurcation diagram for modes of $u ; \varepsilon=0.5,(-+-) \xi=0.01,(-*-) \xi=0.06,(-\nabla-) \xi=0.10$.

Here, $\lambda$ and $\xi$ are the central frequency and bandwidth, respectively. The stationary response variance in the linear case is

$\sigma_{1}^{2}=\frac{\sigma_{f}^{2}}{\omega^{4}} \frac{1-\bar{\lambda}^{2}+(1+\bar{\xi} \bar{\lambda})\left(\bar{\lambda}^{2}+4 \xi \eta \bar{\lambda}\right)}{\left[\left(1-\bar{\lambda}^{2}\right)^{2}+4 \eta \bar{\lambda}(1+\bar{\xi} \bar{\lambda})(\xi+\eta \bar{\lambda})\right]}$,

where

$\bar{\lambda}=\frac{\lambda}{\omega}, \quad \bar{\xi}=\frac{\xi}{\eta}, \quad \sigma_{f}^{2}=\frac{\pi S_{0}}{4 \xi \lambda^{3}}$.

Here again the partially described input is nondimensionalized as $u=\left(f / \omega^{2} \sigma_{1}\right)$, and the frequency parameter is taken as $\delta=\Omega / \lambda$. Solution of Eq. (16) with the above $S_{f f}$ leads to the corresponding $S_{y y}$ for the response process to be Gaussian. Numerical results have been obtained for a system with $\eta=0.08$, $\varepsilon=0.3, \xi=0.02$. In Figs. $4-6$ the response PSD and the input PDF functions are shown corresponding to frequency parameter values of $\bar{\lambda}=1,1.8$ and 3 , respectively. The linear PSD function, which was used as the first approximation for starting the iteration, is also shown in the figures. It is observed that the iteration converges in all the three cases, even though more number of iterations are needed for $\bar{\lambda}=1.8$. The present concept constrains the response to be Gaussian and this makes the solution devoid of energy at certain frequencies as seen from the $S_{y y}$ functions. This exact solution corresponds to non-Gaussian excitations with PDF shown in Figs. 4(b), 5(b) and 6(b). It is interesting to note that the excitation PDF would be bimodal for $\bar{\lambda}=1.8$.

\section{Discussion}

It is shown that for a given excitation PSD, the response of the cubic oscillator can be a Gaussian process, under certain conditions on the PDF of the excitation. These are sufficient conditions and hence there can be other possibilities. Since the response is forced to be Gaussian, knowledge of the PSD function $S_{y y}$ is sufficient to fully describe the PDF of $y(t)$. To find the response PSD, one has to solve Eq. (16) iteratively. This equation reduces to the known solution of the linear system as $\varepsilon$ tends to zero. Thus, in Fig. 3, it is found that for very small non-linearity, the present solution and the exact solution for ideal white noise are close. But, as non-linearity increases, the input has to be strongly non-Gaussian to yield Gaussian response. 
Narrowband excitation brings in new features of the non-linear system. Previously it has been found $[4,5]$ that for narrowband excitations, the response PDF of a cubic oscillator may become bimodal for a certain combination of system parameters. Here this probabilistic bifurcation is seen to occur in the excitation process, as $\lambda / \omega$ is increased from 1 to 1.8 . The modes coalesce again as the value of $\lambda / \omega$ changes to 3 . This behavior depends also on another parameter $\xi$, which controls the bandwidth of the excitation. In Fig. 7, the bifurcation diagram of the modes of the excitation process is shown for three values of $\xi$ as a function of $\lambda / \omega$. Shifting the non-linear term to the right-hand side and treating it as part of the excitation is a mathematical artifice and hence the subsequent results may not be of immediate practical application. Nonetheless, since exact solutions in non-linear random vibration are too rare, the results presented here would be of interest in demonstrating bifurcation of uni-modal PDF to multi-modal PDF. Here this occurs not on the side of the response but on the excitation, owing to the non-linear nature of the system. This in turn makes the response process devoid of energy at certain frequencies. It is interesting to note that this is contrary to the presence of higher harmonics in a non-linear oscillator excited by a fully prescribed Gaussian random process.

\section{Summary}

A new concept has been presented in this paper to investigate a non-linear system under random excitation, partially described in terms of its power spectral density function. The condition under which the response process can be strictly Gaussian is found. Detailed numerical results are presented for a hardening spring cubic oscillator under broad- and narrowband excitations. For the response to be Gaussian, it turns out that the input has to be non-Gaussian. The exact probability structure of this special input is found. However, the condition derived is only sufficient, and hence the solution presented is not unique.

\section{References}

[1] Y.K. Lin, G.Q. Cai, Probabilistic Structural DynamicsAdvanced Theory and Applications, McGraw-Hill, New York, 1995.

[2] J.B. Roberts, P.D. Spanos, Random Vibration and Statistical Linearization, Wiley, Chichester, 1990.

[3] S. Krenk, J.B. Roberts, Local similarity in non-linear random vibration, J. Appl. Mech. 66 (1999) 225-235.

[4] G. Tagata, Analysis of a randomly excited non-linear stretched string, J. Sound Vib. 58 (1) (1978) 95-107.

[5] R.N. Iyengar, Response of non-linear systems to narrow-band excitation, Struct. Safety 6 (1989) $177-185$. 